\title{
Electrostriction in elastic dielectrics undergoing large deformation
}

\author{
Xuanhe Zhao and Zhigang Suo* \\ School of Engineering and Applied Sciences, Harvard University, MA o2138, USA
}

\begin{abstract}
We develop a thermodynamic model of electrostriction for elastic dielectrics capable of large deformation. The model reproduces the classical equations of state for dielectrics at small deformation, but shows that some electrostrictive effects negligible at small deformation may become pronounced at large deformation. The model is then specialized to account for recent experiments with an elastomer, where the electric displacement is linear in the electric field when the strain of the elastomer is held fixed, but the permittivity changes appreciably when the strain changes. Our model couples this quasi-linear dielectric behavior with nonlinear elastic behavior. We explore the practical consequence of the model by deriving conditions under which the deformation-dependent permittivity suppresses electromechanical instability.
\end{abstract}

PACS numbers: $77.65 \cdot-\mathrm{j}, \quad 61.41 .+\mathrm{e}, \quad 77.84 . \mathrm{Jd}$

* email: suo@seas.harvard.edu 


\section{Introduction}

A dielectric deforms when subject to a voltage. ${ }^{1-5}$ The voltage may cause some dielectrics to become thinner, but other dielectrics to become thicker (Fig. 1). The voltage-induced deformation is exploited in diverse applications, including medical equipments, optical devices, energy harvesters, and space robotics. ${ }^{6-9}$ While all dielectrics deform under a voltage, the effect is particularly pronounced in soft materials, such as certain thermoplastic polymers 10 and elastomers $3,{ }^{11-15}$. For example, subject to a voltage a membrane of an elastomer may enlarge many times its area.

For dielectrics that are nonpolar in the absence of electric field, the voltage-induced deformation has been analyzed by invoking stresses of two origins: the electrostrictive stress and the Maxwell stress. The electrostrictive stress results from the effect of deformation on permittivity. Models of electrostriction have long existed, but only for small deformation. ${ }^{16,17}$ For large deformation, almost all existing analyses have only used the Maxwell stress to account for voltage-induced deformation. $3,{ }^{11-13}, 15,18,19$ Maxwell ${ }^{20}$ derived this field of stress in the vacuum to account for electrostatic forces between rigid conductors. For example, for a parallel-plate capacitor, with two oppositely charged electrodes separated by a gap of vacuum, the Maxwell stress describes the electrostatic attraction between the two electrodes.

The Maxwell stress is unable to account for dielectrics that thicken under a voltage (Fig. 1c). Indeed, as we have pointed out in a previous paper ${ }^{21}$, the Maxwell stress can account for voltage-induced deformation only for a very special type of materials, which we call the ideal dielectric elastomers, where the permittivity is deformation-independent. The behavior of the 
ideal dielectric elastomers corresponds to the following physical picture. An elastomer is a crosslinked network of long and flexible polymers. When the degree of crosslink is low and the deformation is well below the extension limit, the molecular units in the polymers can polarize as freely as in a polymeric liquid, so that the permittivity is unaffected by the deformation. However, when the degree of crosslink is not so low, or when the deformation approaches the extension limit, the permittivity of the elastomer will be affected by the deformation. In fact, recent experiments on an elastomer have shown that the permittivity varies by a factor of 2 at large deformation. ${ }^{15}$ Furthermore, there is no reason to assume deformation-independent permittivity for future materials. ${ }^{14,22}$ In such cases, the Maxwell stress by itself is not expected to account for the voltage-induced deformation.

This paper develops a thermodynamic model of electrostriction at large deformation, on the basis of a nonlinear field theory of elastic dielectrics; see Refs [23-25] for reviews. When the permittivity is deformation-dependent, the model shows that the Maxwell stress only contributes to part of the voltage-induced deformation. The model reproduces the classical equations of state for dielectrics at small deformation. To explore the practical consequence of the model, we derive the conditions under which the deformation-dependent permittivity suppresses electromechanical instability.

\section{Work, free-energy function, and equations of state}

To exhibit the essential behavior, we focus on a widely used configuration: a membrane of an elastic dielectric sandwiched between two electrodes (Fig. 2). In the 
undeformed state, the membrane is of sides $L_{1}, L_{2}$ and $L_{3}$. When the dielectric is subject to mechanical forces $F_{1}, F_{2}$ and $F_{3}$ in the three directions, as well as an electric voltage $\Phi$ via an external circuit, the three sides deform to $l_{1}, l_{2}$ and $l_{3}$, and an amount of electric charge $Q$ flows through the external circuit from one electrode to the other. The electrodes are so compliant that they do not constrain the deformation of the dielectric. We neglect the electrostatic energy associated with the fringe field in the vacuum outside the dielectric, and assume that the deformation and electric field inside the dielectric is homogenous.

As noted in our previous paper ${ }^{23}$, we emphasize that $F_{1}, F_{2}$ and $F_{3}$ are mechanical forces applied by external agents, such as weights. We do not invoke the nebulous notion of electric force. When the sides of the membrane change by small amounts, $\delta l_{1}, \delta l_{2}$ and $\delta l_{3}$, the mechanical forces do work $F_{1} \delta l_{1}, F_{2} \delta l_{2}$ and $F_{3} \delta l_{3}$. Similarly, when a small amount of charge, $\delta Q$, relocates from one electrode to the other through the external circuit, the electric voltage does work $\Phi \delta Q$.

The dielectric is a thermodynamic system taken to be held at a constant temperature. Denote the Helmholtz free energy of the system by $A$. When the dielectric is in equilibrium with the applied forces and the applied voltage, associated with any small change in the sides and the induced charge, and change in the Helmholtz free energy equals the work done by the applied forces and the applied voltage, namely,

$$
\delta A=F_{1} \delta l_{1}+F_{2} \delta l_{2}+F_{3} \delta l_{3}+\Phi \delta Q .
$$

Divide (1) by the volume of the dielectric in the reference state, $L_{1} L_{2} L_{3}$, and we obtain that 


$$
\delta W=s_{1} \delta \lambda_{1}+s_{2} \delta \lambda_{2}+s_{3} \delta \lambda_{3}+\widetilde{E} \delta \tilde{D}
$$

where $W=A /\left(L_{1} L_{2} L_{3}\right)$ is the Helmholtz free-energy density, $s_{1}=F_{1} /\left(L_{2} L_{3}\right), s_{2}=F_{2} /\left(L_{1} L_{3}\right)$ and $s_{3}=F_{3} /\left(L_{1} L_{2}\right)$ are the nominal stresses, $\lambda_{1}=l_{1} / L_{1}, \lambda_{2}=l_{2} / L_{2}$ and $\lambda_{3}=l_{3} / L_{3}$ are the stretches, $\widetilde{E}=\Phi / L_{3}$ is the nominal electric field, and $\widetilde{D}=Q /\left(L_{1} L_{2}\right)$ is the nominal electric displacement. It is evident from (2) that the nominal stresses are work-conjugate to the stretches, and the nominal electric field is work-conjugate to the nominal electric displacement.

Following Refs. [21, 23-27], as a material model we stipulate that the free-energy density is a function of the stretches and the nominal electric displacement, $W\left(\lambda_{1}, \lambda_{2}, \lambda_{3}, \tilde{D}\right)$. Consequently, (2) dictates that the nominal stresses and the nominal electric field be the partial differential coefficients, namely,

$$
\begin{gathered}
s_{1}=\frac{\partial W\left(\lambda_{1}, \lambda_{2}, \lambda_{3}, \tilde{D}\right)}{\partial \lambda_{1}}, \\
s_{2}=\frac{\partial W\left(\lambda_{1}, \lambda_{2}, \lambda_{3}, \tilde{D}\right)}{\partial \lambda_{2}}, \\
s_{3}=\frac{\partial W\left(\lambda_{1}, \lambda_{2}, \lambda_{3}, \tilde{D}\right)}{\partial \lambda_{3}}, \\
\widetilde{E}=\frac{\partial W\left(\lambda_{1}, \lambda_{2}, \lambda_{3}, \tilde{D}\right)}{\partial \widetilde{D}} .
\end{gathered}
$$

Once the free-energy function $W\left(\lambda_{1}, \lambda_{2}, \lambda_{3}, \tilde{D}\right)$ is known for a given elastic dielectric, (3) constitutes the equations of state.

Recall that the true stresses are defined as $\sigma_{1}=F_{1} / l_{2} l_{3}, \sigma_{2}=F_{2} / l_{3} l_{1}$ and $\sigma_{3}=F_{3} / l_{1} l_{2}$, so that the true stresses relate to the nominal stresses by $\sigma_{1}=s_{1} /\left(\lambda_{2} \lambda_{3}\right), \sigma_{2}=s_{2} /\left(\lambda_{1} \lambda_{3}\right)$ and 
$\sigma_{3}=s_{3} /\left(\lambda_{1} \lambda_{2}\right)$. Similarly, the true electric field is defined as $E=\Phi / l_{3}$, so that the true electric field relates to the nominal electric field by $E=\widetilde{E} / \lambda_{3}$. Also, the true electric displacement is defined as $D=Q /\left(l_{1} l_{2}\right)$, so that the true electric displacement relates to the nominal electric displacement by $D=\tilde{D} /\left(\lambda_{1} \lambda_{2}\right)$.

While the nominal stress $s_{1}$ is work-conjugate to the stretch $\lambda_{1}$, the true stress $\sigma_{1}$ is not. This statement is understood as follows. The applied force $F_{1}$ does work $F_{1} \delta l_{1}$. This work can be written in terms of the nominal stress:

$$
F_{1} \delta l_{1}=\left(s_{1} L_{2} L_{3}\right) \delta\left(\lambda_{1} L_{1}\right)=\left(L_{1} L_{2} L_{3}\right)\left(s_{1} \delta \lambda_{1}\right) .
$$

Thus, the product $s_{1} \delta \lambda_{1}$ is the work per unit volume, a fact that leads to (3a). By contrast, the same work $F_{1} \delta_{1}$ can also be written in terms of the true stress:

$$
F_{1} \delta l_{1}=\left(\sigma_{1} l_{2} l_{3}\right) \delta\left(\lambda_{1} L_{1}\right)=\left(L_{1} l_{2} l_{3}\right)\left(\sigma_{1} \delta \lambda_{1}\right) .
$$

Thus, the product $\sigma_{1} \delta \lambda_{1}$ is not work per unit volume, and $\sigma_{1} \neq \partial W / \partial \lambda_{1}$.

Analogously, as we noted in our previous paper ${ }^{23}$, while the nominal electric displacement $\widetilde{D}$ is work-conjugate to the nominal electric field $\widetilde{E}$, the true electric displacement $D$ is not work-conjugate to the true electric field $E$. Recall that the applied voltage $\Phi$ does work $\Phi \delta Q$. This work can be written in terms of the nominal quantities:

$$
\Phi \delta Q=\left(\widetilde{E} L_{3}\right) \delta\left(\widetilde{D} L_{1} L_{2}\right)=\left(L_{1} L_{2} L_{3}\right)(\widetilde{E} \delta \tilde{D}) .
$$

Thus, the product $\tilde{E} \delta \tilde{D}$ is the work per unit volume, a fact that leads to (3d). By contrast, the same electric work $\Phi \delta Q$ can also be written in terms of the true quantities:

$$
\Phi \delta Q=\left(E l_{3}\right) \delta\left(D l_{1} l_{2}\right)=\left(l_{1} l_{2} l_{3}\right)(E \delta D)+E D\left(l_{3} l_{1} \delta l_{2}+l_{3} l_{2} \delta l_{1}\right) .
$$

Thus, the product $E \delta D$ is not work per unit volume, and $E \neq \partial W / \partial D$. 
The fact that the true quantities are not work-conjugate makes theoretical arguments using the true quantities often appear very subtle, even when the deformation is small; e.g., Ref. $[16,17]$. As we will show later, such apparent subtleties vanish when we use the nominal quantities. While the merit of the nominal quantities is evident from the work-conjugate relations, i.e. Eq (3), the true quantities seem to appeal to most researchers. In this paper we compromise: we will derive basic relations using the nominal quantities, and then translate these relations in terms of the true quantities.

\section{Quasi-linear dielectrics}

When a dielectric sustains a large deformation and a large electric displacement, the equations of state are nonlinear. The general structure of the nonlinear equations of state for elastic dielectrics has been reviewed recently. ${ }^{23-25}$ This section considers a special type of dielectric behavior, which we call quasi-linear dielectric behavior, where the electric displacement is linear in the electric field when the strain is held fixed, but the permittivity may vary when the strain changes. This quasi-liner behavior seems to describe the experimental observation of many elastomers. ${ }^{3,11-13,15,18,19}$ The quasi-linear dielectrics include ideal dielectric elastomers as a special case. For the latter, the electric field is linear in the electric displacement, and the permittivity is deformation-independent. Furthermore, as we will show below, the quasi-linear dielectric model naturally extends the model of small-strain electrostriction widely used in the literature. ${ }^{16,17}$

To account for the quasi-linear dielectric behavior, we expand the free-energy function 
$W\left(\lambda_{1}, \lambda_{2}, \lambda_{3}, \tilde{D}\right)$ into the Taylor series in terms of $\tilde{D}$ up to the quadratic term:

$$
W\left(\lambda_{1}, \lambda_{2}, \lambda_{3}, \tilde{D}\right)=W_{S}\left(\lambda_{1}, \lambda_{2}, \lambda_{3}\right)+\frac{1}{2} \beta\left(\lambda_{1}, \lambda_{2}, \lambda_{3}\right) \widetilde{D}^{2} .
$$

The leading term $W_{S}\left(\lambda_{1}, \lambda_{2}, \lambda_{3}\right)$ is the elastic energy in the absence of the applied voltage. The material is taken to be nonpolar, so that by symmetry the term linear in $\widetilde{D}$ vanishes. The coefficient of the quadratic term, $\beta$, is a function of the stretches.

Inserting (8) into (3d), we obtain that

$$
\widetilde{E}=\beta \tilde{D}
$$

This equation characterizes the quasi-linear dielectric: the electric field is linear in the electric displacement when the stretches are held at any fixed levels. In terms of true quantities, (9) becomes $\lambda_{3} E=\beta \lambda_{1} \lambda_{2} D$. We recover the familiar equation $D=\varepsilon E$ by identifying $\varepsilon=\lambda_{3} /\left(\lambda_{1} \lambda_{2} \beta\right)$. In general, the permittivity $\varepsilon$ is a function of the stretches, $\varepsilon\left(\lambda_{1}, \lambda_{2}, \lambda_{3}\right)$.

Using the permittivity, we rewrite (8) as

$$
W\left(\lambda_{1}, \lambda_{2}, \lambda_{3}, \tilde{D}\right)=W_{S}\left(\lambda_{1}, \lambda_{2}, \lambda_{3}\right)+\frac{\lambda_{1}^{-1} \lambda_{2}^{-1} \lambda_{3}}{2 \varepsilon\left(\lambda_{1}, \lambda_{2}, \lambda_{3}\right)} \tilde{D}^{2}
$$

Inserting (10) into (3), we obtain that

$$
\begin{gathered}
s_{1}=\frac{\partial W_{S}}{\partial \lambda_{1}}-\frac{1}{2 \varepsilon} \lambda_{1}^{-2} \lambda_{2}^{-1} \lambda_{3} \widetilde{D}^{2}-\frac{1}{2 \varepsilon^{2}} \frac{\partial \varepsilon}{\partial \lambda_{1}} \lambda_{1}^{-1} \lambda_{2}^{-1} \lambda_{3} \widetilde{D}^{2} \\
s_{2}=\frac{\partial W_{S}}{\partial \lambda_{2}}-\frac{1}{2 \varepsilon} \lambda_{1}^{-1} \lambda_{2}^{-2} \lambda_{3} \widetilde{D}^{2}-\frac{1}{2 \varepsilon^{2}} \frac{\partial \varepsilon}{\partial \lambda_{2}} \lambda_{1}^{-1} \lambda_{2}^{-1} \lambda_{3} \widetilde{D}^{2} \\
s_{3}=\frac{\partial W_{S}}{\partial \lambda_{3}}+\frac{1}{2 \varepsilon} \lambda_{1}^{-1} \lambda_{2}^{-1} \widetilde{D}^{2}-\frac{1}{2 \varepsilon^{2}} \frac{\partial \varepsilon}{\partial \lambda_{3}} \lambda_{1}^{-1} \lambda_{2}^{-1} \lambda_{3} \widetilde{D}^{2} \\
\widetilde{E}=\frac{1}{\varepsilon} \lambda_{1}^{-1} \lambda_{2}^{-1} \lambda_{3} \tilde{D}
\end{gathered}
$$

In terms of the true quantities, (11) becomes that 


$$
\begin{gathered}
\sigma_{1}=\lambda_{2}^{-1} \lambda_{3}^{-1} \frac{\partial W_{S}}{\partial \lambda_{1}}-\frac{\varepsilon}{2} E^{2}-\frac{1}{2} \frac{\partial \varepsilon}{\partial \lambda_{1}} \lambda_{1} E^{2} \\
\sigma_{2}=\lambda_{1}^{-1} \lambda_{3}^{-1} \frac{\partial W_{S}}{\partial \lambda_{2}}-\frac{\varepsilon}{2} E^{2}-\frac{1}{2} \frac{\partial \varepsilon}{\partial \lambda_{2}} \lambda_{2} E^{2} \\
\sigma_{3}=\lambda_{1}^{-1} \lambda_{2}^{-1} \frac{\partial W_{S}}{\partial \lambda_{3}}+\frac{\varepsilon}{2} E^{2}-\frac{1}{2} \frac{\partial \varepsilon}{\partial \lambda_{3}} \lambda_{3} E^{2} \\
D=\varepsilon E .
\end{gathered}
$$

Once the two functions $W_{S}\left(\lambda_{1}, \lambda_{2}, \lambda_{3}\right)$ and $\varepsilon\left(\lambda_{1}, \lambda_{2}, \lambda_{3}\right)$ are known for a quasi-linear dielectric, (12) constitutes the equations of state. Eq. (12d) recovers the familiar relation for a linear dielectric when the stretches are held fixed. Eqs. (12a-c) have a similar form. As an example, in what follows we discuss (12c). The first term in (12c) is the stress due to elasticity, and can be either tensile or compressive. The second term is the Maxwell stress, and is always tensile in the direction of the electric field. The third term is present when the permittivity varies with the stretch, and can be either tensile or compressive. Observe that the third term in (12c) also scales with $\lambda_{3}$, which differs greatly from unity when the dielectric deforms substantially. Consequently, some electrostrictive effect negligible at small deformation may become significant at large deformation.

As noted before, the Maxwell stress can fully account for the voltage-induced stress only when the permittivity is independent of deformation. When the permittivity is deformation-dependent, the third term in (12c) can either add to the tensile Maxwell stress when $\partial \varepsilon / \partial \lambda_{3}<0$, or reduce the effect of the tensile Maxwell stress when $\partial \varepsilon / \partial \lambda_{3}>0$. Recall that a voltage can cause some dielectrics to become thinner, but other dielectrics to become 
thicker (Fig. 1).

A few remarks on terminology may be helpful. In (12c), the contribution from the electric field separates into two terms: one term scales with $\mathcal{E}$, and the other scales with $\partial \varepsilon / \partial \lambda_{3}$. It seems natural to call the former the Maxwell stress, and the latter the electrostrictive stress. Such a separation is possible only for quasi-linear dielectrics, for which the permittivity is defined. For a generally nonlinear dielectric, however, the equations of state are given by (3), and there may not be a natural way to single out part of the stress and call it the Maxwell stress. In such a case, one may as well call the whole voltage-induced deformation electrostriction, and refrain from the temptation to divide the deformation in some artificial manner.

\section{Small-strain approximation}

Past analyses of electrostriction have assumed small deformation. ${ }^{16,}{ }^{17}$ The resulting equations of state are sometimes used without justification for elastomers at large deformation. ${ }^{28,29}$ To contrast the equations of state at small and large deformation, here we specialize our results for large deformation to those for small deformation.

When deformation is small, all three stretches are close to the unity, $\lambda_{i} \approx 1$. Consequently, one can expand the function $\varepsilon\left(\lambda_{1}, \lambda_{2}, \lambda_{3}\right)$ into the Taylor series up to terms linear in $\left(\lambda_{i}-1\right)$, namely,

$$
\varepsilon=\bar{\varepsilon}\left[1+a\left(\lambda_{3}-1\right)+b\left(\lambda_{1}+\lambda_{2}+\lambda_{3}-3\right)\right]
$$

where $\bar{\varepsilon}$ is the permittivity of the dielectric in the absence of deformation, and $a$ and $b$ are the 
coefficients of electrostriction. ${ }^{16,17}$ In writing (13), we have assumed that the dielectric is isotropic. When the electric field is applied in direction 3, by symmetry, the coefficient of electrostriction is the same in directions 1 and 2, but is different in direction 3 .

Inserting (13) into (12), we obtain that

$$
\begin{gathered}
\sigma_{1}=\lambda_{2}^{-1} \lambda_{3}^{-1} \frac{\partial W_{S}}{\partial \lambda_{1}}-\frac{1}{2} \bar{\varepsilon}\left[1+a\left(\lambda_{3}-1\right)+b\left(2 \lambda_{1}+\lambda_{2}+\lambda_{3}-3\right)\right] E^{2} \\
\sigma_{2}=\lambda_{1}^{-1} \lambda_{3}^{-1} \frac{\partial W_{S}}{\partial \lambda_{2}}-\frac{1}{2} \bar{\varepsilon}\left[1+a\left(\lambda_{3}-1\right)+b\left(\lambda_{1}+2 \lambda_{2}+\lambda_{3}-3\right)\right] E^{2} \\
\sigma_{3}=\lambda_{1}^{-1} \lambda_{2}^{-1} \frac{\partial W_{S}}{\partial \lambda_{3}}+\frac{1}{2} \bar{\varepsilon}\left[1-a+b\left(\lambda_{1}+\lambda_{2}-3\right)\right] E^{2} \\
D=\bar{\varepsilon}\left[1+a\left(\lambda_{3}-1\right)+b\left(\lambda_{1}+\lambda_{2}+\lambda_{3}-3\right)\right] E
\end{gathered}
$$

In the small-strain approximation, $\lambda_{i}-1$ are small compared to the unity, and the coefficients of electrostriction $a$ and $b$ are assumed to be of order unity, so that (14) becomes

$$
\begin{gathered}
\sigma_{1}=\frac{\partial W_{S}}{\partial \lambda_{1}}-\frac{1}{2} \bar{\varepsilon}(1+b) E^{2}, \\
\sigma_{2}=\frac{\partial W_{S}}{\partial \lambda_{2}}-\frac{1}{2} \bar{\varepsilon}(1+b) E^{2}, \\
\sigma_{3}=\frac{\partial W_{S}}{\partial \lambda_{3}}+\frac{1}{2} \bar{\varepsilon}(1-a-b) E^{2} . \\
D=\bar{\varepsilon} E .
\end{gathered}
$$

Eqs. (15a-d) agree with the classical results in Refs. [16, 17]. This agreement is not accidental. Although the derivation of the existing model takes a different approach from ours, both derivations are based on the same assumption: quasi-linear dielectrics at small deformation. The classical results, however, are restricted to small deformation. At large deformation, one 
should use more general equations of state, (3) or (12).

\section{Incompressible elastic dielectrics}

When an elastomer deforms, the change in shape is usually much more pronounced than the change in volume. Consequently, it is a common practice to assume that the elastomer is incompressible. This section derives the equations of state for incompressible, quasi-linear dielectrics.

The condition of incompressibility places a constraint among the three stretches: $\lambda_{1} \lambda_{2} \lambda_{3}=1$, so that when the membrane deforms, the change $\delta \lambda_{3}$ relates to the changes $\delta \lambda_{1}$ and $\delta \lambda_{2}$ as

$$
\delta \lambda_{3}=-\frac{\delta \lambda_{1}}{\lambda_{1}^{2} \lambda_{2}}-\frac{\delta \lambda_{2}}{\lambda_{2}^{2} \lambda_{1}}
$$

Consequently, (2) becomes

$$
\delta W=\left(s_{1}-\frac{s_{3}}{\lambda_{1}^{2} \lambda_{2}}\right) \delta \lambda_{1}+\left(s_{2}-\frac{s_{3}}{\lambda_{2}^{2} \lambda_{1}}\right) \delta \lambda_{2}+\widetilde{E} \delta \tilde{D}
$$

As a material model, we stipulate that the free-energy density is a function of the two in-plane stretches and the nominal electric displacement, $W\left(\lambda_{1}, \lambda_{2}, \tilde{D}\right)$, so that (17) implies that

$$
\begin{gathered}
s_{1}-\frac{s_{3}}{\lambda_{1}^{2} \lambda_{2}}=\frac{\partial W\left(\lambda_{1}, \lambda_{2}, \tilde{D}\right)}{\partial \lambda_{1}}, \\
s_{2}-\frac{s_{3}}{\lambda_{2}^{2} \lambda_{1}}=\frac{\partial W\left(\lambda_{1}, \lambda_{2}, \tilde{D}\right)}{\partial \lambda_{2}}, \\
\widetilde{E}=\frac{\partial W\left(\lambda_{1}, \lambda_{2}, \tilde{D}\right)}{\partial \widetilde{D}} .
\end{gathered}
$$


Once the free-energy function $W\left(\lambda_{1}, \lambda_{2}, \tilde{D}\right)$ is known for an incompressible elastic dielectric, (18) constitutes the equations of state.

For an incompressible, quasi-linear dielectric, the free-energy function is specialized from (10) and takes the form

$$
W\left(\lambda_{1}, \lambda_{2}, \tilde{D}\right)=W_{S}\left(\lambda_{1}, \lambda_{2}\right)+\frac{\lambda_{1}^{-2} \lambda_{2}^{-2}}{2 \varepsilon\left(\lambda_{1}, \lambda_{2}\right)} \widetilde{D}^{2}
$$

As indicated, both the elastic energy density $W_{S}$ and the permittivity $\varepsilon$ are functions of the two in-plane stretches. Inserting (19) into (18), we obtain that

$$
\begin{gathered}
s_{1}-\frac{s_{3}}{\lambda_{1}^{2} \lambda_{2}}=\frac{\partial W_{S}}{\partial \lambda_{1}}-\frac{1}{\varepsilon} \lambda_{1}^{-3} \lambda_{2}^{-2} \widetilde{D}^{2}-\frac{1}{2 \varepsilon^{2}} \frac{\partial \varepsilon}{\partial \lambda_{1}} \lambda_{1}^{-2} \lambda_{2}^{-2} \widetilde{D}^{2}, \\
s_{2}-\frac{s_{3}}{\lambda_{2}^{2} \lambda_{1}}=\frac{\partial W_{S}}{\partial \lambda_{2}}-\frac{1}{\varepsilon} \lambda_{1}^{-2} \lambda_{2}^{-3} \widetilde{D}^{2}-\frac{1}{2 \varepsilon^{2}} \frac{\partial \varepsilon}{\partial \lambda_{2}} \lambda_{1}^{-2} \lambda_{2}^{-2} \widetilde{D}^{2}, \\
\widetilde{E}=\frac{\lambda_{1}^{-2} \lambda_{2}^{-2}}{\varepsilon} \widetilde{D} .
\end{gathered}
$$

In terms of true quantities, (20) becomes

$$
\begin{gathered}
\sigma_{1}-\sigma_{3}=\lambda_{1} \frac{\partial W_{S}}{\partial \lambda_{1}}-\varepsilon E^{2}-\frac{1}{2} \frac{\partial \varepsilon}{\partial \lambda_{1}} \lambda_{1} E^{2} \\
\sigma_{2}-\sigma_{3}=\lambda_{2} \frac{\partial W_{S}}{\partial \lambda_{2}}-\varepsilon E^{2}-\frac{1}{2} \frac{\partial \varepsilon}{\partial \lambda_{2}} \lambda_{2} E^{2} \\
D=\varepsilon E
\end{gathered}
$$

Once the two functions $W_{S}\left(\lambda_{1}, \lambda_{2}\right)$ and $\varepsilon\left(\lambda_{1}, \lambda_{2}\right)$ are known for an incompressible, quasi-linear dielectric, (21) constitutes the equations of state.

In recent experiments, Wissler and Mazza stretched membranes of a VHB elastomer by an equal amount in the two in-plane directions, and measured the permittivity as a function of 
the stretch. ${ }^{15}$ As shown in Fig. 3, we fit their experimental data to the following function:

$$
\varepsilon\left(\lambda_{1}, \lambda_{2}\right)=\bar{\varepsilon}\left[1+c\left(\lambda_{1}+\lambda_{2}-2\right)\right]
$$

with $c=-0.053$ and $\bar{\varepsilon}=4.68 \varepsilon_{0}$, where $\varepsilon_{0}=8.85 \times 10^{-12} \mathrm{~F} / \mathrm{m}$ is the permittivity of the vacuum.

By substituting Eq. (22) into (21), we obtain that

$$
\begin{aligned}
& \sigma_{1}-\sigma_{3}=-\bar{\varepsilon}\left[1+c\left(\frac{3}{2} \lambda_{1}+\lambda_{2}-2\right)\right] E^{2}, \\
& \sigma_{2}-\sigma_{3}=-\bar{\varepsilon}\left[1+c\left(\lambda_{1}+\frac{3}{2} \lambda_{2}-2\right)\right] E^{2} .
\end{aligned}
$$

In writing (23), we have only retained the contributions due to the electric field, and dropped those due to elasticity. Eq. (23a) is plotted in Fig 4 using the experimental value of the coefficient of electrostriction, $c=-0.053$. For comparison, Fig. 4 also includes several expressions sometimes used in the literature: the Maxwell stress with constant permittivity 3, 11-13, ${ }_{15}, 18,19,-\bar{\varepsilon} E^{2}$; the Maxwell stress with varying permittivity ${ }^{15},-\bar{\varepsilon}\left[1+c\left(\lambda_{1}+\lambda_{2}-2\right)\right] E^{2}$; and electrical stress from small strain electrostriction ${ }^{28,29},-\bar{\varepsilon}(1+c / 2) E^{2}$. At small deformation $\left(\lambda_{1}=\lambda_{2} \approx 1\right)$, the electrical stress of other forms are close to the prediction of our model. However, as deformation increases, the electrical stresses of all the other expressions significantly deviate from our prediction. At $\lambda_{1}=\lambda_{2}=6$, the deviation of the Maxwell stress with constant permittivity and small-strain electrostriction are around $70 \%$, and the deviation of the Maxwell stress with varying permittivity is more than 30\%. Dielectric elastomer usually works at a pre-stretched state $\left(\lambda_{1}, \lambda_{2}=3 \sim 6\right)$, and gives a very high actuation strain (over 100\%). The large deformation may cause significant errors in almost all previous models on dielectric 
elastomers.

\section{Electromechanical instability}

Subject to a voltage, a dielectric elastomer reduces its thickness, so that the voltage induces a high electric field. The positive feedback between the electric field and the thickness may cause the elastomer to thin down drastically, resulting in an electrical breakdown. This electromechanical instability was first described by Stark and Garton ${ }^{15}$, and has been studied for ideal dielectric elastomers ${ }^{21,26,30}$. In this section, we examine the effect of deformation-dependent permittivity on the electromechanical instability, using a method developed in Refs. [21, 26].

We will consider the case where the elastomer is subject to no mechanical force, $s_{1}=s_{2}=s_{3}=0$, and is subject to a voltage. By symmetry, the two in-plane stretches are equal, which we denote as $\lambda_{1}=\lambda_{2}=\lambda$. Due to incompressibility, the stretch in the direction of thickness is give by $\lambda_{3}=\lambda^{-2}$. We write the free-energy function as

$$
W(\lambda, \tilde{D})=\frac{\mu}{2}\left(2 \lambda^{2}+\lambda^{-4}-3\right)+\frac{\lambda^{-4} \widetilde{D}^{2}}{2 \bar{\varepsilon}[1+2 c(\lambda-1)]}
$$

In writing (24), we have used the deformation-dependent permittivity (22), and assumed that the elastomer is a network of long and flexible polymers obeying the Gaussian statistics, with $\mu$ being the shear modulus. ${ }^{31}$ As the results below will indicate, when the electromechanical instability does occur, the critical stretch is modest. For such a modest level of stretch, the Gaussian statistics is expected to describe the elasticity of the elastomer adequately. 
Equation (20c) becomes

$$
\frac{\widetilde{E}}{\sqrt{\mu / \bar{\varepsilon}}}=\frac{\lambda^{-4}}{1+2 c(\lambda-1)} \frac{\tilde{D}}{\sqrt{\bar{\varepsilon} \mu}}
$$

In the absence of the applied force, the voltage causes the elastomer to expand in the plane, so that $\lambda$ also depends on $\widetilde{D}$, and $\widetilde{E}$ is no longer linear in $\widetilde{D}$. Indeed, the electromechanical instability sets in when the function $\widetilde{E}(\widetilde{D})$ reaches maximum. ${ }^{21,26}$

We next construct the nonlinear function $\tilde{E}(\tilde{D})$. The condition $s_{1}(\lambda, \tilde{D})=\mathrm{o}$ implies that $\partial W(\lambda, \widetilde{D}) / \partial \lambda=0$. Inserting (24) into $\partial W(\lambda, \widetilde{D}) / \partial \lambda=0$, we obtain that

$$
\frac{\tilde{D}}{\sqrt{\bar{\varepsilon} \mu}}=\frac{2\left(\lambda^{6}-1\right)[1+2 c(\lambda-1)]}{2+c \lambda[1+2 c(\lambda-1)]^{-1}}
$$

We can regard the stretch $\lambda$ as the independent variable, and calculate $\widetilde{D}$ from (26), and then calculate $\widetilde{E}$ from (25).

Fig 5 plots (25) and (26) in several ways, using various values of the coefficient of electrostriction, c. The critical points for electromechanical instability are marked by crosses. For $c=0$, the permittivity is independent of deformation, and the results recover those for the ideal dielectric elastomers. ${ }^{26}$ For $c=-0.053$, the nominal electric field reaches peak at the stretch $\lambda \approx 1.28$. For $c=1$, the nominal electric field reaches peak at a smaller stretch, $\lambda \approx 1.18$.

When $c<0$, the effect of deformation-dependent permittivity partially removes the Maxwell stress. Consequently, a sufficiently negative coefficient of electrostriction $c$ will suppress electromechanical instability. When $c=-10$, for example, the elastomer becomes thicker under applied voltage, a fact that eliminates the positive feedback between the true 
electric field and the thickness, so that the dielectric is electromechanically stable. When $c=-0.25$, even though the elastomer becomes thinner with the applied voltage, the deformation-dependent permittivity can still suppress electromechanical instability.

In addition to the electromechanical instability, the elastomer may also fail by electrical breakdown. Fig. 5c shows that the true electric field $E$ is a monotonic function of $\widetilde{D}$. The true electric field is useful to estimate the condition of electrical breakdown.

Fig. 6 plots the critical conditions for electromechanical instability $\left(\widetilde{E}_{c}, E_{c}\right.$ and $\left.\lambda_{c}\right)$ as a function of the coefficient of electrostriction, $c$. As $c$ decreases, the critical values of the true electric field, nominal electrical field, and stretch all increase. When $c<-0.23$, electromechanical instability is suppressed.

\section{Concluding remarks}

Models of electrostriction have long existed, but only for small deformation. This paper develops a model of electrostriction that is consistent with thermodynamics at large deformation. Motivated by recent experiments on dielectric elastomers, our model couples nonlinear elastic behavior and quasi-linear dielectric behavior. The model shows that electrostrictive effects negligible at small deformation may become pronounced at large deformation. Based on experimentally determined permittivity-stretch function for VHB, we calculate the voltage-induced stress using several available models. We show that the stress predicted by the previous models markedly deviate from that predicted by the present model. Our model also has implications for designing new materials. For example, we derive conditions 
under which the deformation-dependent permittivity suppresses electromechanical instability.

\section{Acknowledgements}

This research was supported by the DARPA through a program on Programmable Matter, and by the National Science Foundation through the MRSEC at Harvard University. XHZ acknowledges the support of the Founder's Prize, of the American Academy of Mechanics, sponsored by the Robert M. and Mary Haythornthwaite Foundation. 


\section{References}

1 R. E. Newnham, Properties of Materials (Oxford University Press 2005, Oxford, 2005).

$2 \quad$ J. Su, T. B. Xu, S. J. Zhang, T. R. Shrout, and Q. M. Zhang, Applied Physics Letters 85, 1045 (2004).

3 R. Pelrine, R. Kornbluh, Q. B. Pei, and J. Joseph, Science 287, 836 (2000).

4 Q. M. Zhang, V. Bharti, and X. Zhao, Science 280, 2101 (1998).

$5 \quad$ K. Uchino, S. Nomura, L. E. Cross, S. J. Jang, and R. E. Newnham, Journal of Applied Physics 51, 1142 (1980).

6 A. Wingert, M. D. Lichter, and S. Dubowsky, Ieee-Asme Transactions on Mechatronics 11, 448 (2006).

7 Y. M. Liu, K. L. Ren, H. F. Hofmann, and Q. M. Zhang, Ieee Transactions on Ultrasonics Ferroelectrics and Frequency Control 52, 2411 (2005).

8 J. Q. Xia, Y. R. Ying, and S. H. Foulger, Advanced Materials 17, 2463 (2005).

$9 \quad$ Y. Bar-Cohen, Journal of Spacecraft and Rockets 39, 822 (2002).

$10 \quad$ K. H. Stark and C. G. Garton, Nature 176, 1225 (1955).

11 R. E. Pelrine, R. D. Kornbluh, and J. P. Joseph, Sensors and Actuators a-Physical 64, 77 (1998).

12 G. Kofod, P. Sommer-Larsen, R. Kronbluh, and R. Pelrine, Journal of Intelligent Material Systems and Structures 14, 787 (2003).

13 J. S. Plante and S. Dubowsky, International Journal of Solids and Structures 43, 7727 (2006).

14 S. M. Ha, W. Yuan, Q. B. Pei, R. Pelrine, and S. Stanford, Advanced Materials 18, 887 (2006).

$15 \quad$ M. Wissler and E. Mazza, Sensors and Actuators a-Physical 138, 384 (2007).

16 J. A. Stratton, Electromagnetic Theory (McGraw-Hill, New York, 1941).

17 L. D. Landau and E. M. Lifshitz, Electrodynamics of Continuous Media (Pergamon, New York, 1984).

18 F. Carpi and D. De Rossi, Materials Science \& Engineering C-Biomimetic and Supramolecular Systems 24, 555 (2004).

19 E. M. Mockensturm and N. Goulbourne, International Journal of Non-Linear Mechanics 41, 388 (2006).

J. C. Maxwell, A treatise on electricity and magnetism (Clarendon Press, Oxford 1998). 
28

T. Yamwong, A. M. Voice, and G. R. Davies, Journal of Applied Physics 91, 1472 (2002).

R. Trujillo, J. Mou, P. E. Phelan, and D. S. Chau, International journal of advanced manufacturing technology 23, 176 (2004).

A. N. Norris, Applied Physics Letters 92, 2 (2008).

L. R. G. Treloar, The Physics of Rubber Elasticity (Clarendon Press, Oxford, 1975). 


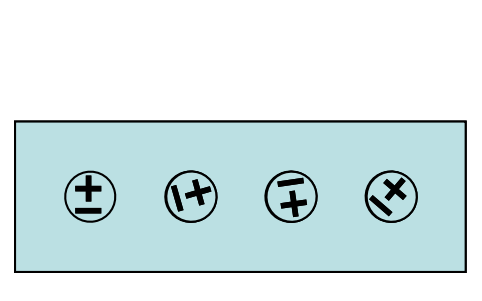

(a)

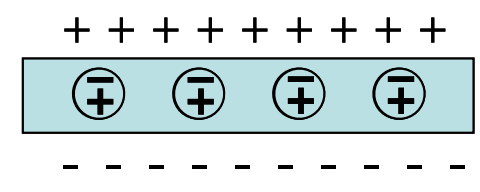

(b)

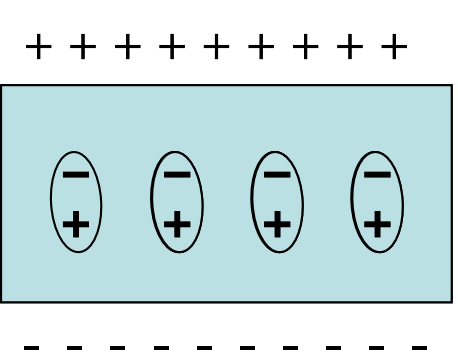

(c)

FIG. 1. Consider a dielectric nonpolar in the absence of applied voltage (a). Subject to a voltage, some dielectrics become thinner (b), but other dielectrics become thicker (c). 


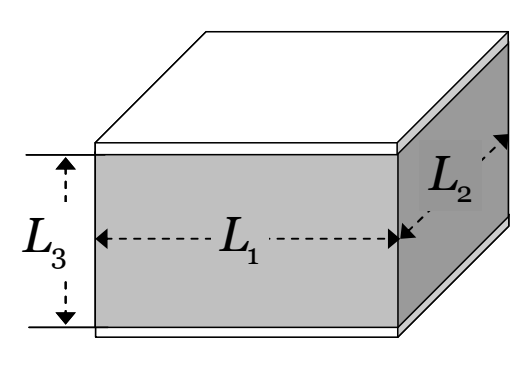

(a)

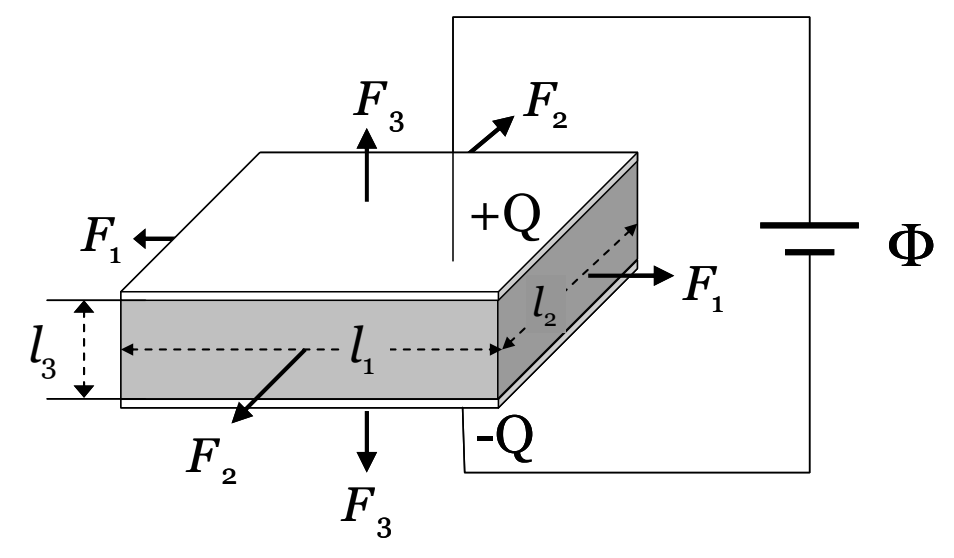

(b)

FIG. 2. A membrane of an elastic dielectric is sandwiched between two compliant electrodes.

(a) Under no applied forces and voltage, the membrane is of sides $L_{1}, L_{2}$ and $L_{3}$. (b) Subject to mechanical forces $F_{1}, F_{2}$ and $F_{3}$ in three directions, and to electrical voltage $\Phi$ via an external circuit, the membrane deforms to $l_{1}, l_{2}$ and $l_{3}$, and charge $Q$ flows from one electrode to the other through the external circuit. 


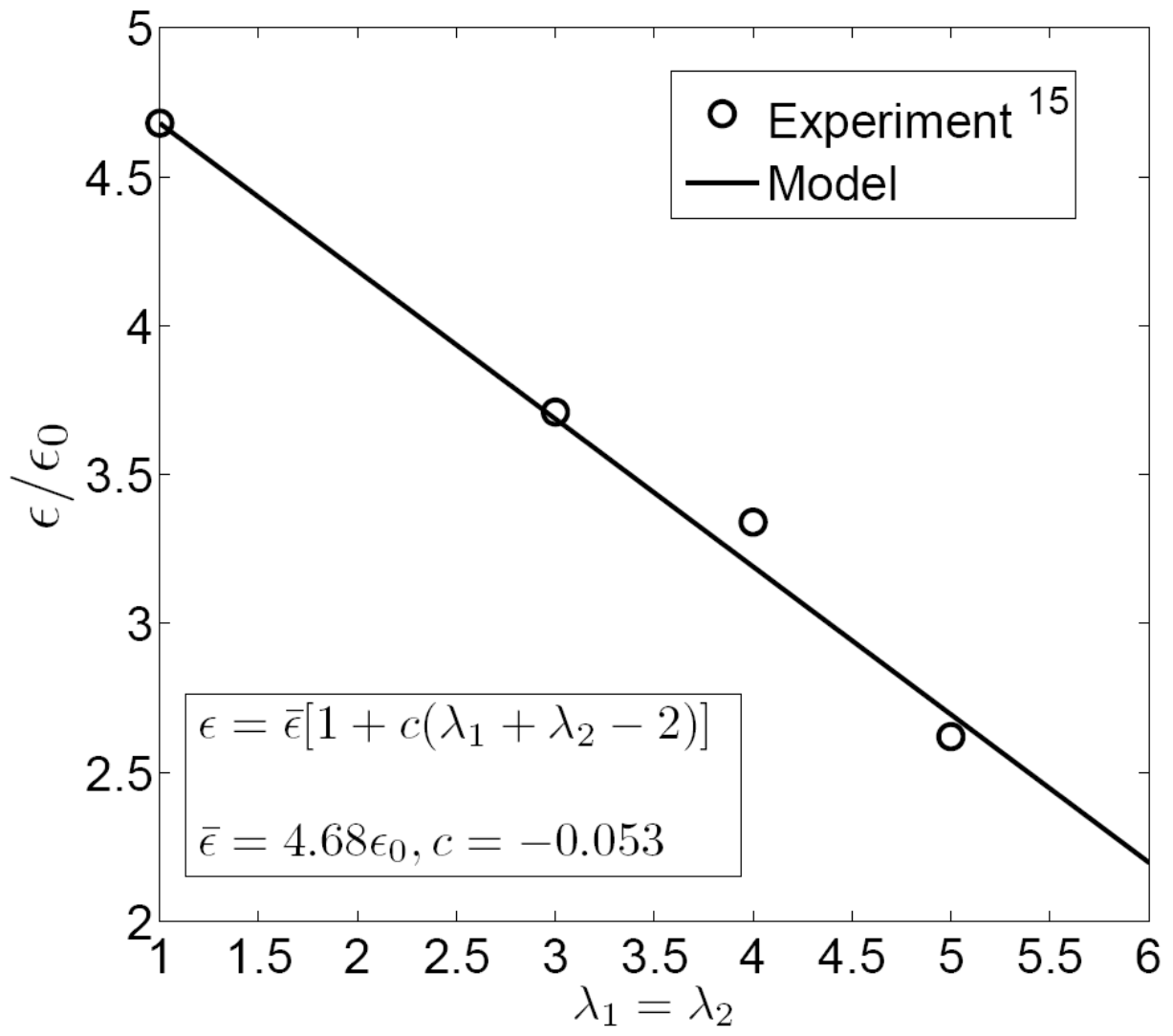

FIG. 3. Experimentally measured permittivity as a function of deformation. ${ }^{15}$ The experimental data are fitted to a straight line with $\bar{\varepsilon}=4.68 \varepsilon_{\mathrm{o}}$ and $c=-0.053$, where $\varepsilon_{\mathrm{o}}=8.85 \times 10^{-12} \mathrm{~F} / \mathrm{m}$ is the permittivity of vacuum. 


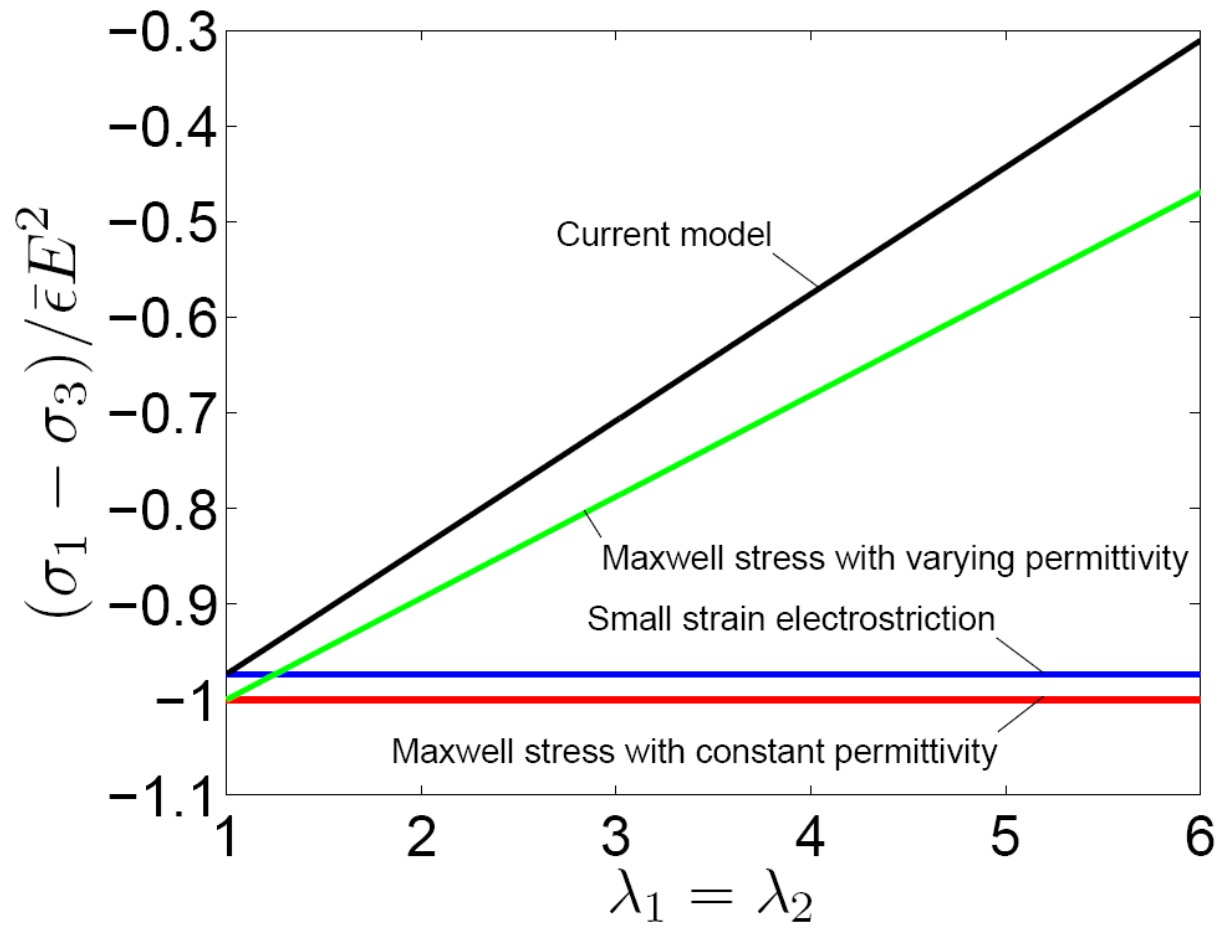

FIG. 4. Comparison of stresses calculated using various methods. Only the voltage-induced stresses are plotted. 

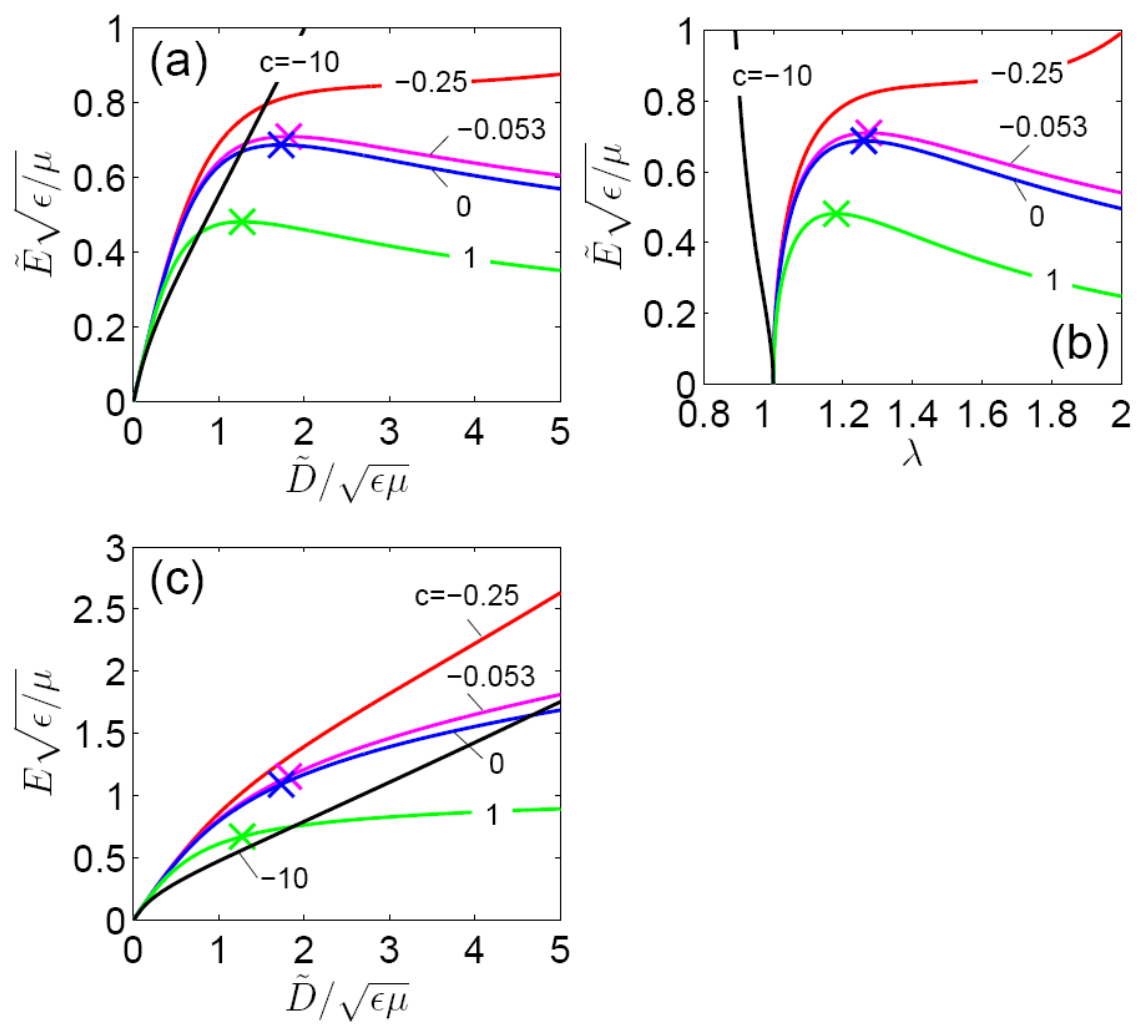

FIG. 5. Behavior of dielectric elastomers for various values of the coefficient of electrostriction. (a) nominal electric field vs nominal electric displacement, (b) true electric field vs nominal electric displacement, and (c) nominal electric field vs actuation stretch. The critical points for electromechanical instability are marked by crosses. 

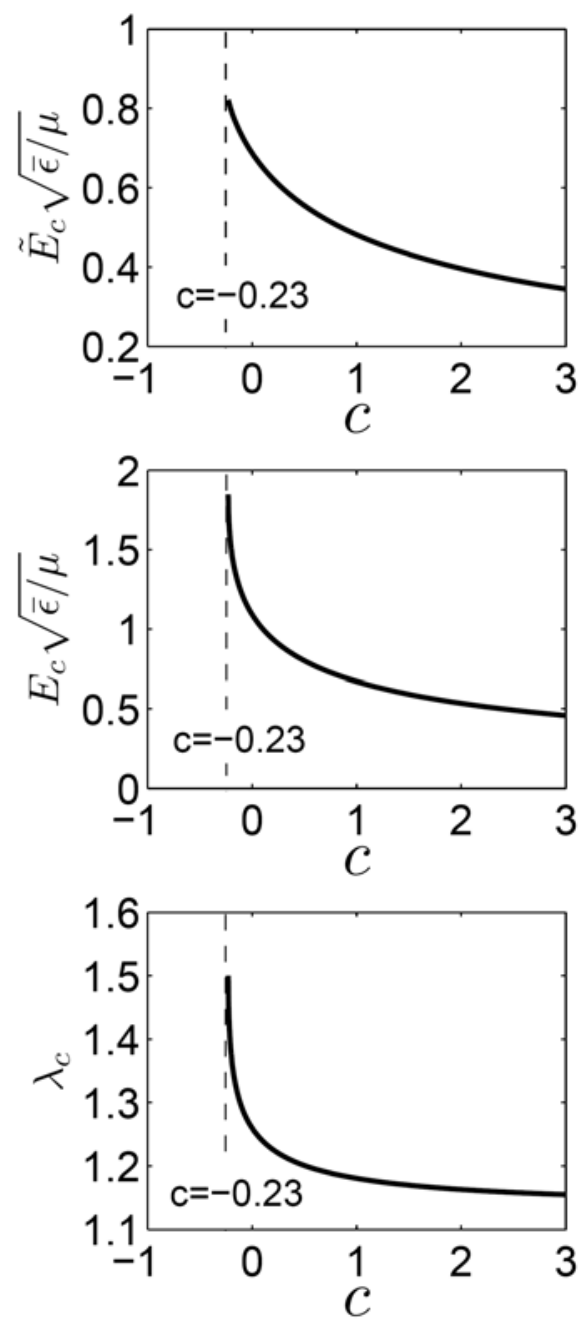

FIG. 6. The effect of the coefficient of electrostriction on electromechanical instability. As $c$ decreases, the critical true electric field, nominal electrical field, and stretch for electromechanical instability all increase. When $c<-0.23$, electromechanical instability is suppressed. 